\title{
A Survey on Emotion Recognition from EEG Signals for Autism Spectrum Disorder
}

\author{
N. Mohanapriya \\ Assistant Professor, \\ Department of Computer \\ Science and Engineering, \\ Vivekanandha College of \\ Engineering for Women, \\ Namakkal, Tamilnadu, India
}

\author{
L. Malathi, PhD \\ Associate Professor \\ Department of Computer \\ Science and Engineering, \\ Vivekanandha College of \\ Engineering for Women, \\ Namakkal, Tamilnadu, India
}

\author{
B. Revathi \\ PG Scholar, \\ Department of Computer \\ Science and Engineering, \\ Vivekanandha College of \\ Engineering for Women, \\ Namakkal, Tamilnadu, India
}

\begin{abstract}
Children with Autism Spectrum Disorder (ASD) cannot express their emotions explicitly; this makes it difficult for the parents and caretakers associated with these children to understand the child's behavior, leading to a major setback in the child's early developmental stages. To identify the autism for child initial stages can help early diagnosis. Delayed detection of child autism leads to incurable. This paper analysis the existing works on detection of autism spectrum disorder from EEG signal. Various filtering technique and classification are presented. The experiment for were conducted for support vector machine (SVM), k-nearest neighbor (KNN), linear discriminant analysis (LDA), deep learning, Naive Bayes, Random Forest, deep-learning classification algorithms. Here the deep learning algorithm gives better results for autism recognition with the emotions such as happy, calm, anger and scared. As the no of medical records increases the conventional techniques is not suitable for handle large number data.
\end{abstract}

\section{Keywords}

EEG Signal, Emotion Recognition, Autism Spectrum Disorder, Deep Learning, classification

\section{INTRODUCTION}

Autism is a neuron developmental disorder characterized by impaired social interaction, impaired verbal and non-verbal communication, and restricted and repetitive behavior. Parents usually notice signs in the first two years of their child's life. These signs often develop gradually, though some children with autism reach their developmental milestones at a normal pace and then regress. The diagnostic criteria require that symptoms become apparent in early childhood, typically before age three.

Emotion plays an important role in our daily life and work. In this paper focused on emotion recognition from human brain activity and measured by EEG signals. Electroencephalography (EEG) is an electrophysiological monitoring method to record electrical activity of the brain. An emotion is a mental and physiological state associated with a wide variety of feelings, thoughts, and behavior. An emotion is a subjective experience which makes studying emotions one of the most confused and still open fields of research in psychology. Deep learning is part of a broader family of machine learning methods based on learning data representations, as proposed to task-specific algorithms. Learning can be supervised, partially supervised or unsupervised.

\section{LITERATURE REVIEW}

In [1] authors Niranjana Krupa, Karthik Anantharam, Manoj Sanker, Sameer Datta and John Vijay Sagar in this paper "Recognition of emotions in autistic children using physiological signals" The protocols used for acquiring signals from the autistic children and details of the total number of samples recorded are explained. A systematic and an efficient protocol were followed during the data acquisition phase. Only the children in the age group of 3 to 12 were considered for data acquisition. The samples from Autistic children needed for the research were acquired at the Department of Child and Adolescent Psychiatry (CAP) at the National Institute of Mental Health and Neuro Sciences (NIMHANS), Bangalore, India and Academy for Severe Handicaps and Autism (ASHA), Bangalore, India. The initial pilot study was carried out at SHA. After the successful completion of our pilot study we received an approval by the Ethical Review Board of NIMHANS to carry out the proposed study at NIMHANS for the duration of six months. Samples from control subjects were recorded from children who belong to the same age group as those of children with Autism. The whole data acquisition process has been carried out only after obtaining informed consent

A wearable wristband for acquire physiological signal and an algorithm, using a support vector machine (SVM) classifier, that will calculate emotional states such as neutral, happy \& interest of children with autism. A support vector machine classifier wires the recognition of the three emotions on neutral, happy and interest in children among autism. Determining the particular emotional status from the physiological answer is a classification problem somewhere the attribute are the physiological features extract from the physiological signals mention above and the goal function is the particular emotional status being qualified. This paper discusses a non-invasive, safe method to be aware of and map emotions in children through Autism. Robust systems comprise of a wearable wristband embedded with sensors to acquire data and a classification algorithm to recognize emotions was considered and developed successfully, with accuracy of good in later age children, and inefficient for less than three years child.

In [2] authors K. G. Smitha, A. P. Vinod in this paper "Facial emotion recognition system for autistic children: a feasible study based on FPGA implementation" Children through autism spectrum disorder have complexity in understanding the emotional and mental state starting the facial expression of the people they interact. The inability to recognize other people's emotion wills setback their interpersonal 
communication. The portability of the system resolve makes positive ease of use and real-time emotion recognition and that determination help for direct feedback while communicate with caretaker. Principal component analysis (PCA) has been recognized as the least complex quality extraction algorithm to be implementing in hardware. In this paper, we present an in depth study of the performance of serial and parallel implementation of PCA in order to identify the most possible method for recognition of a portable emotion detector for autism children. In this paper, we have compare two hardware-efficient implementations. Jacobi's iteration which is the parallel implementation is completed hardware efficient by calculating only the diagonal and upper triangular matrix by exploit the symmetry property of the covariance matrix. We have compared the PCA of the two future emotion detectors along with the implementation existing in the same word length of 8 bits. In this paper, we provide a evaluation between serial and parallel method of principal component analysis method in order to realize which among them will be the best method for recognition of a moveable emotion detector for autism children.

In [3] authors Felix Albu, Daniela Hagiescu, Liviu Vladutu, Mihaela-Alexandra Puica in this paper "neural network approaches for children's emotion Recognition in intelligent learning applications" The feature vector is formed with the following parameters the short term energy, the zero-crossing rate, the spectral roll-off, spectral centroid and $13 \mathrm{Mel}$ Frequency Costrel Coefficients. The first element is the short term energy. This parameter is computed for each frame of 10 $\mathrm{ms}$ of speech. It is widely used for silence periods detection and audio classes discrimination. The energy of voiced frames is higher than that of unvoiced frames. The spectral roll-off is a measure of the spectral shape and it is defined as the frequency below which $80 \%$ of the magnitude distribution is concentrated. The Dartmouth Database of Children Faces, which contains thousands of images taken for 100 children ( 50 boys and 50 girls) ages 5 to 15 years old showing 8 facial expressions: anger, disgust, fear, happy, neutral, pleased, sad and surprise. The first step is to detect the landmarks on children's faces, thus distinguishing the elements of interest on the face: eyes, eyebrows and mouth. By muscle contraction, shape of these elements and distance between them changes for different emotional states. The differences are small, there can be seen that a negative expression has a smaller mouth and closer eyebrows and a positive expression has a wider mouth and eyebrows are spread apart

The children emotion recognition performance of several neural networks approach is described. The Radial Basis Function (RBF), Probabilistic Neural Networks (PNN) and Support Vector Machines (SVM) variant were tested on record speech signals and face detected images. For the language sign the MFCC and other parameter were computed in concert with their mean and standard difference in order to obtain the feature vector for the neural network input. For image input parameters for emotion detection consisted in several distance computed between certain facial appearance using space coordinate for eyes, eyebrows and lips.

They have shown similar performance to RBF network in our emotion detection simulation. In this paper we evaluate several network structures for an emotion recognition application. The emotion recognition is based on record speech signals and face detected images. And investigate the robustness of the classification rate to clamor on both speech and image signals. Next to be focused on correlating the results using images and speech and improve the results by selecting the most suitable features for the chosen network architecture. It is used to several neural networks techniques and Doesn't work well for images and speech signals.

In [4] authors Paul Fergus, Basma Abdulaimma, Chris Carter, Sheena Round "Interactive Mobile Technology for Children with Autism Spectrum Condition (ASC)" Mobile Computing has been gradually more used to support children with autism. These higher technologies with their associated features provide a comfortable and inexpensive solution for autistic children and their family. The portability, flexibility and availability of features have opened up new opportunity for enhancing and inspiring the quality of support to ease autistic children deficiency. Autistic children are known for their destruction in understanding and interpreting the emotional facial expression. This paper posits a 3D animation solution develop for mobile device. The animation has been built to the desires of children with autism. Facial animations have been implemented with warning to improve the major difficulty in understanding and recognizing emotions in facial expressions.

In [5] authors Keiran M. Rump, Joyce L. Giovannelli, Nancy J. Minshew , Mark S. Strauss in this paper "The Development of Emotion Recognition in Individuals With Autism" Emotion recognition was investigated in typically developing individuals and individuals with autism. Experiment tested children with brief video displays of facial expressions that varied in subtlety. Whereas the performance of control individuals was best in the adult group, the performance of individuals with autism was similar in all age groups. Results are discussed with respect to underlying cognitive processes that may be affecting the development of emotion recognition in individuals with autism. This was the first study to look at the ability to recognize facial expressions in young children through to adults in both typically developing individuals and individuals with autism. In contrast to prior research, this study controlled for the subtlety of the expressions and required participants make judgments of only briefly presented dynamic expressions.

In [6] authors Raja Majid Mehmood, Ruoyu Du 2 and Hyo Jong Lee "Optimal Feature Selection And Deep Learning Ensembles Method For Emotion Recognition From Human Brain EEG Sensors" In this paper to recognize emotional state by analyzing the skin texture of electroencephalography (EEG) signals, which are generated from EEG sensor that non-invasively measure the electrical activity of neurons inside the human brain, and select the optimal mixture of these features for recognition. The Hjorth parameters activity, mobility, and complexity were used to measure the signal activity of the time run data. We selected the optimal EEG features using a balanced one-way ANOVA after calculate the Hjorth parameter for different frequency ranges. The optimal features are further processed for emotion categorization using support vector machine (SVM), linear discriminant analysis (LDA), k-nearest neighbor (KNN), Naïve Bayes, Random Forest, deep-learning. The categorization process was performed using Waikato Environment for Knowledge Analysis (WEKA) software. All selected classifiers were trained and tested over the group of four emotions. We used the default setting available in WEKA for both classifier. In this paper, we proposed an EEG feature extraction and selection method for emotion happy, calm, sad, and scared recognition. To select the optimal feature set, we analyze the extract feature set using a balanced one-way ANOVA (pvalue $<0.05$ ) method. To further improve emotion recognition performance, we need to explore additional feature 
combination with more emotional program in the arousalvalence domain.

In [7] authors Yongbin Gaol, Hyo Jong Lee, Raja Majid Mehmood in this paper "Deep Learning Of EEG Signals For Emotion Recognition" EEG-based emotion research is in a preliminary stage. A total of 21 subjects (12 male and 9 female) participated in this experiment. All subjects were students at the same institute and were from 12 to 14 years old. The selected subjects were first informed of the purpose of our study and the experimental procedure. The subjects were then given a brief introduction and completed consent forms. After the recorded EEG data of each subject were verified, the EEG signals were preprocessed using the EEGLAB toolbox provided by SCCN Lab. The main preprocessing steps consisted of artifact rejection, filtering, and epoch selection from the raw EEG data. The EEG signals were recorded using the Emotiv-EPOC system. This device has a maximum of 16 electrodes and collects 128 samples every second. We used 14 electrodes to record the neural activity of the brain. In addition, the common mode sense (CMS) active electrode and driven right leg (DRL) passive electrode were attached on the mastoid bone behind the ears to create an average reference channel (CMS/DRL). Tomography has shown that each brain region has a different simultaneous emotional response, so decided to record all EEG channels.

In this paper to recognize emotional state by analyzing the skin texture of electroencephalography (EEG) signals, which are generated from EEG sensor that non-invasively measure the electrical activity of neurons inside the human brain, and select the optimal mixture of these features for recognition. The Hjorth parameters activity, mobility, and complexity were used to measure the signal activity of the time run data. We selected the optimal EEG features using a balanced oneway ANOVA after calculate the Hjorth parameter for different frequency ranges. The optimal features are further processed for emotion categorization using support vector machine (SVM), linear discriminant analysis (LDA), knearest neighbor (KNN), Naïve Bayes, Random Forest, deeplearning.

The categorization process was performed using Waikato Environment for Knowledge Analysis (WEKA) software. All selected classifiers were trained and tested over the group of four emotions. We used the default setting available in WEKA for both classifier. In this paper, we proposed an EEG feature extraction and selection method for emotion happy, calm, sad, and scared recognition. To select the optimal feature set, we analyze the extract feature set using a balanced one-way ANOVA (p-value < 0.05) method. To further improve emotion recognition performance, we need to explore additional feature combination with more emotional program in the arousal-valence domain. In this method the emotion recognition is improved but used less no of features used.

In [8], Emotion Recognition from Face Dataset Using Deep Neural Nets, This paper is facial emotion recognition using face dataset consisting of four classes of emotions happy, angry, neutral and sad with different models of deep neural networks. The raw pixels values of all images in CMU face images dataset. The pixels values were are represented by higher level concepts in Restricted Boltzmann Machine, Deep Belief Networks and Stacked Auto encoder. This model could learn to recognize emotion with considerably higher accuracy compare two models. We have used the CMU face image dataset which consists of 640 black and white face images. Each images consisted in pose, emotional expression, size and eyes. RBM is hidden layer and visible layer. The DBN contains two hidden layers and a visible layer. The Deep learning blocks such as RBM, DBN and SAE+SM learn the representations formed by the pixels and predict the existence of emotion. The results show that the SAE+SM could learn to recognize facial emotions with an accuracy of $99.68 \%$ with only 19 hidden nodes in both the auto encoders. However, even by varying the no hidden nodes in hidden layers of RBM and DBN in the range $[50,3000]$ in steps of 50 , the recognition accuracy did not exceed $25.52 \%$. Therefore, we conclude that RBM and DBM could be good in feature extraction, but they are not good in feature classification. On the other hand, SAE+SM is good in feature extraction as well as feature classification.

C. Petrantonakis, et.al., [9], Emotion Recognition from Brain Signals Using Hybrid Adaptive Filtering and Higher Order Crossings Analysis, A feature extraction method for a userindependent emotion recognition system, namely, HAF-HOC, from electroencephalograms (EEGs). A novel filtering procedure, namely, Hybrid Adaptive Filtering (HAF), for an efficient extraction of the emotion-related EEG-characteristics was developed by applying Genetic Algorithms to the Empirical Mode Decomposition-based representation of EEG signals. In addition, Higher Order Crossings (HOCs) analysis was employed for feature extraction realization from the HAF-filtered signals. The introduced HAF-HOC scheme incorporated four different classification methods to accomplish a robust emotion recognition performance. EEG data is six basic emotions are happiness, surprise, anger, fear, disgust and sadness have been acquired from 16 healthy subjects using three EEG channels.

Experimental results from the application of the HAF-HOC to the collected EEG data and comparison with previous approaches have shown that the HAF-HOC scheme clearly surpasses the latter in the field of emotion recognition from brain signals. This work combined HAF with HOCs analysis to initially process the EEG signals for enhancing the underlying emotion information, by incorporating EMD and GA-based techniques and then applying a feature extraction analysis that resulted in a HOC-based feature vector with increased classification potential. The best results were obtained using SVM for the hardest case of differentiating among the six basic emotions. The comparison of the introduced HAF-HOC method with other relevant feature extraction and emotion recognition robustness and consistency to more effectively discriminate emotions from EEG signals. It achieved maximum classification but emotion effective is less.

In [10] authors Pascal Ackermann, Christian Kohlschei_, Jo' $A^{\prime}$ gila Bitsch, Klaus Wehrle and Sabina Jeschke in this paper "EEG-based Automatic Emotion Recognition: Feature Extraction, Selection and Classification Methods" Automatic emotion recognition is an interdisciplinary research field which deals with the algorithmic detection of human affect evaluation of human emotion is often done using oral feedback or questionnaires during doctor-patient sessions. EEG channel locations and frequency bands are best suited for is an issue of ongoing research. Here to evaluate the use of state of the art feature extraction, feature selection and classification algorithms for EEG emotion classification using data from the de facto standard dataset. This work for Support Vector Machines (SVM) and Random Forests (RF) are applied as two very different state of classification algorithms which are trained using features algorithm are Short-time Fourier Transform (STFT), Higher Order Crossing (HOC) and 
Hilbert-Huang Spectrum (HHS). STFT-based features were obtained from the original signal which is being internally decomposed. HOC features were additionally retrieved from the original signal. Random Forests are also applied for classification which is a representative of the feature selection class of embedded methods meaning that these kinds of classification algorithms.

In [11] authors Aravind E Vijayan, Deepak Sen, Sudheer A.P in this paper "EEG-based Emotion Recognition using Statistical measures and Auto-regressive modeling" A novel approach towards classification of various human emotions based on statistically weighed autoregressive modeling of Electroencephalogram (EEG) signals is discussed. This algorithm was proven to be greater to many correlated works, in distinctive different emotions such as happiness, fear, sadness. The conclusion discussed is based on the results obtained using scale emotion based EEG database called DEAP. This is extracted from data using numerical measures such as Shannon Entropy and advanced order auto-regressive model was well to extract features. The model was used for classify human emotion. The algorithm proposed was tested on a target multimodal dataset prepared by Queen Mary University of London for the taxonomy of human affective states. This dataset commonly recognized as the DEAP dataset is prepared by recording EEG signals of 32 participants though watching 40 different kinds of music videos. The present work can be additional extended in different ways such as online EEG data acquirement using custom-made wearable EEG headset, explore on the optimization of the proposed algorithm. Different perspectives towards emotion representation and it is not conceivable that a practical SOM-based face recognition system.

\section{Table 1. Comparison Analysis of Emotion Recognition Algorithms}

\begin{tabular}{|c|c|c|c|c|}
\hline S. No & Title & Algorithm & Merits & Demerits \\
\hline 1 & $\begin{array}{l}\text { Recognition of emotions in autistic } \\
\text { children using physiological signals }\end{array}$ & Support Vector Machine & $\begin{array}{l}\text { Accuracy is good in } \\
\text { later age children }\end{array}$ & $\begin{array}{l}\text { Inefficient for less } \\
\text { than three years } \\
\text { child. }\end{array}$ \\
\hline 2 & $\begin{array}{l}\text { Facial emotion recognition system } \\
\text { for autistic children: a feasible study } \\
\text { based on FPGA implementation }\end{array}$ & Principal component analysis & $\begin{array}{l}\text { hardware-efficient of } \\
\text { emotion } \\
\text { recognition } \\
\text { architectures is } \\
\text { implemented }\end{array}$ & Low accuracy \\
\hline 3 & $\begin{array}{l}\text { Neural network approaches for } \\
\text { children's emotion Recognition in } \\
\text { intelligent learning applications }\end{array}$ & $\begin{array}{l}\text { 1.Radial Basis Function } \\
\text { 2.Support Vector Machine } \\
\text { 3.Probabilistic Neural } \\
\text { Networks }\end{array}$ & $\begin{array}{l}\text { used to several neural } \\
\text { networks techniques }\end{array}$ & $\begin{array}{l}\text { Doesn't work well } \\
\text { for images and } \\
\text { speech signals }\end{array}$ \\
\hline 4 & $\begin{array}{l}\text { Interactive Mobile Technology for } \\
\text { Children with Autism Spectrum } \\
\text { Condition (ASC) } \\
\end{array}$ & Autism Spectrum Condition & $\begin{array}{l}\text { Produce well-validated } \\
\text { and high-quality works }\end{array}$ & $\begin{array}{l}\text { In efficient for real } \\
\text { time applications }\end{array}$ \\
\hline 5 & $\begin{array}{l}\text { The Development of Emotion } \\
\text { Recognition in Individuals With } \\
\text { Autism }\end{array}$ & Analysis of variance & $\begin{array}{l}\text { Hidden notes used this } \\
\text { algorithm }\end{array}$ & Accuracy is very low \\
\hline 6 & $\begin{array}{l}\text { Optimal feature selection and Deep } \\
\text { Learning Ensembles Method for } \\
\text { emotion recognition from human } \\
\text { brain EEG sensors }\end{array}$ & $\begin{array}{l}\text { 1.Support vector machine } \\
\text { 2.k-nearest neighbors } \\
\text { 3. linear discriminant } \\
\text { analysis, 4. Naïve Bayes } \\
\text { 5. Random Forest }\end{array}$ & $\begin{array}{l}\text { Emotion recognition is } \\
\text { improved }\end{array}$ & $\begin{array}{l}\text { Here less no of } \\
\text { features used }\end{array}$ \\
\hline 7 & $\begin{array}{l}\text { Deep Learning Of EEG Signals For } \\
\text { Emotion Recognition }\end{array}$ & $\begin{array}{l}\text { 1.Support vector machine } \\
\text { 2.k-nearest neighbors } \\
\text { 3.artifical neural network } \\
\end{array}$ & Produce more accuracy & $\begin{array}{l}\text { supports for } \\
\text { detection of other } \\
\text { emotion }\end{array}$ \\
\hline 8 & $\begin{array}{l}\text { Emotion Recognition from Face } \\
\text { Dataset Using Deep Neural Nets }\end{array}$ & $\begin{array}{l}\text { 1.Restricted Boltzmann } \\
\text { Machine } \\
\text { 2. Deep Belief Networks }\end{array}$ & $\begin{array}{l}\text { Hidden nodes in this } \\
\text { method used to increase } \\
\text { performance }\end{array}$ & $\begin{array}{l}\text { Feature classification } \\
\text { is not efficient in this } \\
\text { algorithm }\end{array}$ \\
\hline 9 & $\begin{array}{l}\text { Emotion Recognition from Brain } \\
\text { Signals Using Hybrid Adaptive } \\
\text { Filtering and Higher Order } \\
\text { Crossings Analysis }\end{array}$ & $\begin{array}{l}\text { 1. Quadratic Discriminant } \\
\text { Analysis } \\
\text { 2. k-Nearest Neighbor } \\
\text { 3. Support Vector Machine } \\
\text { 4. Mahalanobis Distance }\end{array}$ & $\begin{array}{l}\text { Achieved maximum } \\
\text { classification }\end{array}$ & $\begin{array}{l}\text { Emotion effective is } \\
\text { less }\end{array}$ \\
\hline 10 & $\begin{array}{l}\text { EEG-based Automatic Emotion } \\
\text { Recognition: Feature Extraction, } \\
\text { Selection and Classification } \\
\text { Methods }\end{array}$ & $\begin{array}{l}\text { 1.Support vector machine } \\
\text { 2.random forest } \\
\text { 3.ranked multipliable rule }\end{array}$ & $\begin{array}{l}\text { Produce well-validated } \\
\text { and high-quality works }\end{array}$ & $\begin{array}{l}\text { It is not set of } \\
\text { electrodes to identify } \\
\text { specific emotions }\end{array}$ \\
\hline
\end{tabular}


Table 2: Accuracy of ASD algorithms

\begin{tabular}{|c|c|}
\hline Algorithm & Accuracy (\%) \\
\hline SVM & $90.0 \%$ \\
\hline RBFNN & $83.06 \%$ \\
\hline PNN & $80.58 \%$ \\
\hline KNN & $83.06 \%$ \\
\hline Deep learning & $92.1 \%$ \\
\hline
\end{tabular}

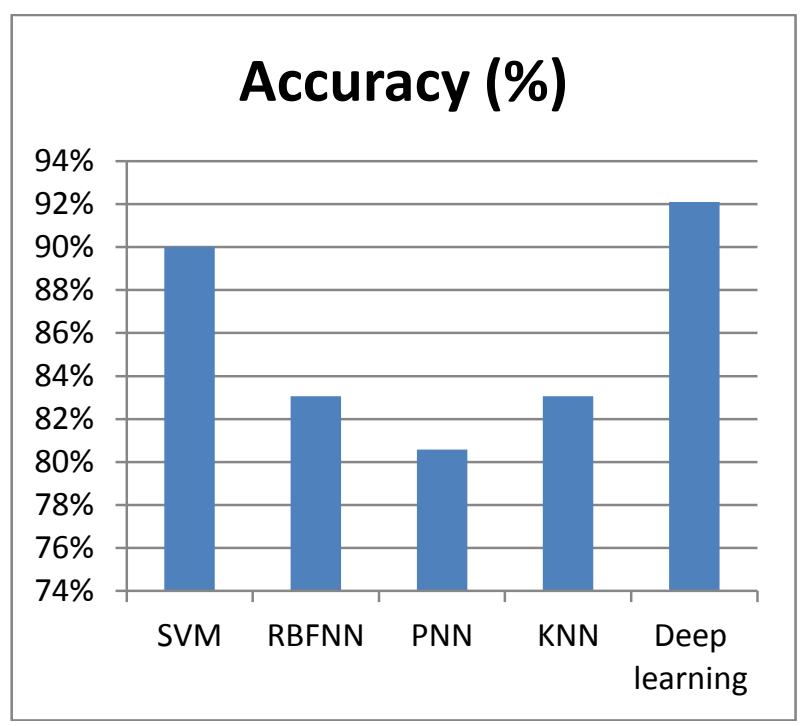

Figure 1. Accuracy Comparison

Table 3: Comparison Analysis of Emotion Classification

\begin{tabular}{|c|c|c|}
\hline Algorithm & Emotions & Accuracy (\%) \\
\hline SVM & happy, calm, sad, and scared & $84.25 \%$ \\
\hline KNN & Happy, sad, love and anger & $60.8 \%$ \\
\hline ANN & happy, calm, sad, and scared & $60 \%$ \\
\hline DBN & happy, anger, neutral and sad & $88.24 \%$ \\
\hline QDA & happiness, surprise, anger, fear, disgust, sadness & $76.6 \%$ \\
\hline
\end{tabular}

Table 3: Comparison

\begin{tabular}{|c|c|}
\hline Algorithm & Sensitivity \\
\hline ML-SVM & $70 \%$ \\
\hline NB & $70.9 \%$ \\
\hline RF & $50 \%$ \\
\hline PNN & $58.75 \%$ \\
\hline
\end{tabular}

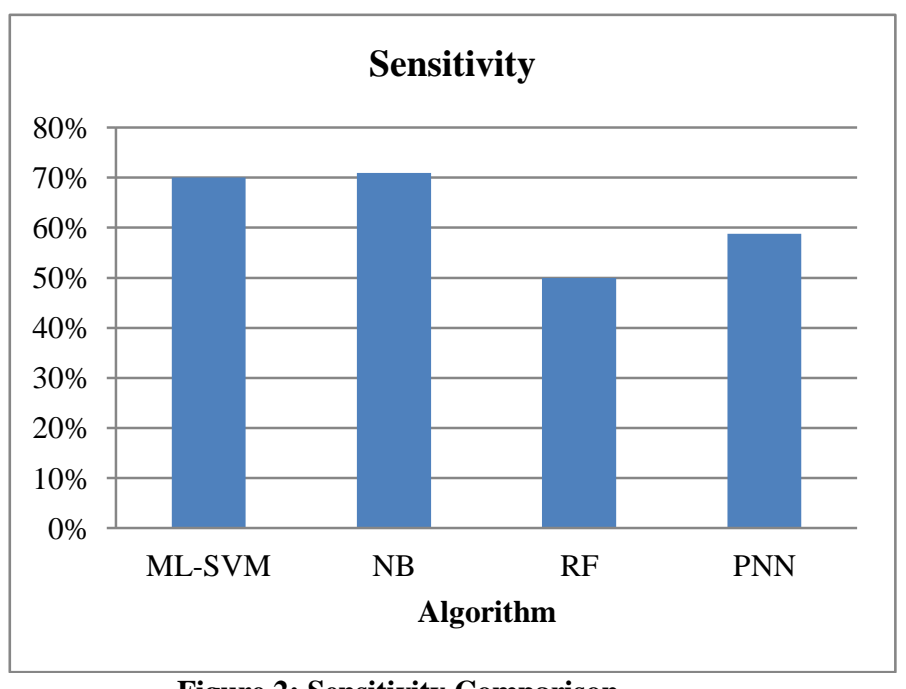

Figure 2: Sensitivity Comparison 
Table 4: Comparison Analysis of Specificity

\begin{tabular}{|c|c|}
\hline Algorithm & Specificity \\
\hline SVM & $60 \%$ \\
\hline LR & $71.30 \%$ \\
\hline QDA & $53.00 \%$ \\
\hline RMR & $65.34 \%$ \\
\hline
\end{tabular}

\section{CONCLUSION}

In this paper, presented different frequency bands, EEG channel locations and feature extraction algorithms for their suitability in EEG-based emotion recognition. Various feature extraction algorithms were applied. The features were found to be valuable feature extraction algorithms for classifying EEG data according to emotions felt. Further we showed the increased importance of features and EEG locations corresponding to the pre frontal and left temporal lobe for EEG emotion classification which coincides with findings from neuroscience and related work. SVM, linear discriminant analysis, random forest, naïve Bayes, decision making algorithms are applied on the EEG dataset and the deep learning given better accuracy for finding the artesian child with the sad, happy, angry and scared emotions.

\section{REFERENCES}

[1] Niranjana Krupa, Karthik Anantharam, Manoj Sanker Sameer Datta John Vijay Sagar, "Recognition of emotions in autistic children uses physiological signals", in Received: 21 July 2015 /Accepted: 8 March 2016.

[2] K. G. Smitha A. P. Vinod, "Facial emotion recognition system for autistic children: a feasible study based on FPGA implementation", International Federation for Medical and Biological Engineering 2015.

[3] Felix Albu, Daniela Hagiescu, Liviu Vladutu, MihaelaAlexandra Puica, "Neural Network Approaches For Children's Emotion Recognition In Intelligent Learning Applications", Proceedings of edulearn15 Conference 6th-8th July 2015.

[4] Paul Fergus, Basma Abdulaimma, Chris Carter, Sheena Round, "Interactive Mobile Technology for Children with Autism Spectrum Condition (ASC)", in 2014.

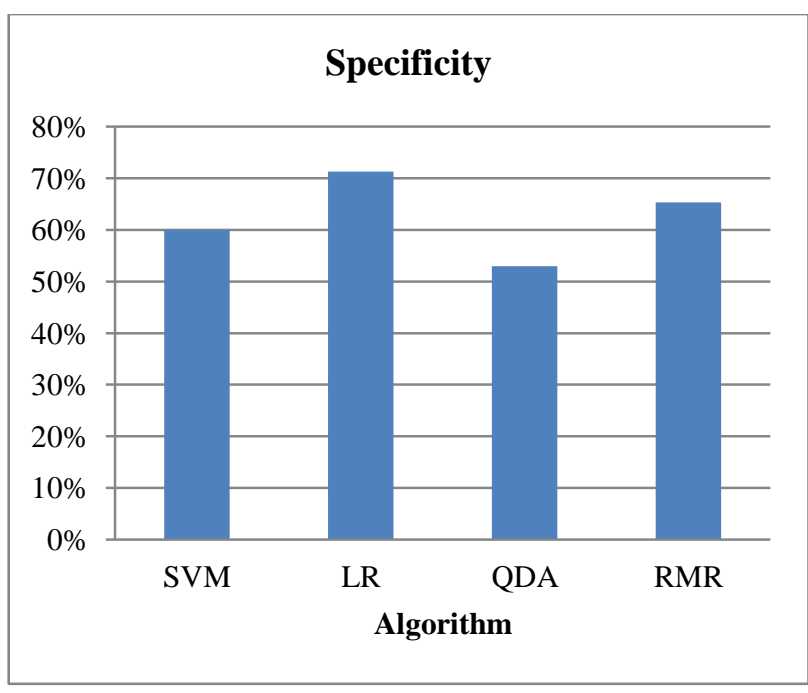

Figure 3: Specificity Comparison

[5] Keiran M. Rump, Joyce L. Giovannelli, Nancy J. Minshew, Mark S. Strauss, "The Development of Emotion Recognition in Individuals with Autism", Child Development, September/October 2009, Volume 80, Number 5, Pages 1434-1447.

[6] Raja Majid Mehmood, Ruoyu Du and Hyo Jong Lee, "Optimal feature selection and Deep Learning Ensembles Method for emotion recognition from human brain EEG sensors", journal of Citation information: DOI 10.1109/ACCESS.2017.2724555, IEEE.

[7] Yongbin Gaol, Hyo Jong Lee, Raja Majid Mehmood, "Deep Learning Of EEG Signals For Emotion Recognition", in IEEE International Conference, 2015.

[8] Olga Sourina, Yisi Liu, "A Fractal-Based Algorithm of Motion Recognition from EEG Using Arousal-Alence ModeL", in 2011.

[9] Panagiotis C. Petrantonakis, Leontios J. Hadjileontiadis, "Emotion Recognition from Brain Signals Using Hybrid Adaptive Filtering and Higher Order Crossings Analysis", IEEE transactions on affective computing, vol. 1 , no. $2,2010$.

[10] Pascal Ackermann, Christian Kohlschein, Jo' A' gila Bitschx, Klaus Wehrlex and Sabina Jeschke, "EEGbased Automatic Emotion Recognition: Feature Extraction, Selection and Classification Methods", in InternationalConference, 2016

[11] Aravind E Vijayan, Deepak Sen, Sudheer A.P "EEGbased Emotion Recognition using Statistical measures and Auto-regressive modeling", 2015 IEEE International Conference on Computational Intelligence \& Communication Technology, 978-1-4799-6023-1/15 $\$ 31.00$ @ 2015 IEEE. 